\title{
Artificial Intelligence and the Challenge for Rural Medicine
}

\author{
James Denvir, PhD ${ }^{1}$
}

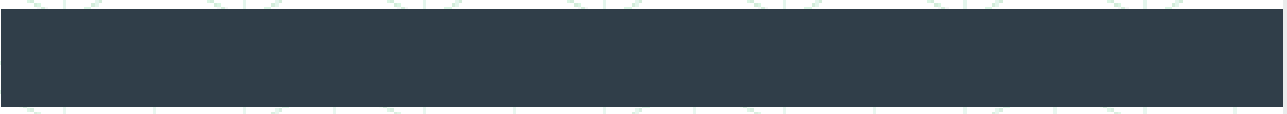

Author affiliations are

listed at the end of this

article.

Correspondence to:

James Denvir, PhD

Marshall Unviersity

Joan C. Edwards

School of Medicine

denvir@marshall.edu

\section{KEYWORDS}

Artificial Intelligence, Rural Medicine, Genomics, Radiology, Pathology

The past three decades have seen major advances in the field of artificial intelligence (AI), specifically in the specializations machine learning and deep learning. In the current decade, those advances have found applications that have brought the field to public consciousness; these applications include image and face recognition and close to fully autonomous vehicles.

Medical science, with its traditional emphasis on the doctor-patient relationship, has been understandably slower in the adoption of these technological advances. However, in his recent review article, ${ }^{1}$ Eric Topol catalogs recent applications of $\mathrm{Al}$ and deep learning to medicine, and in his book "Deep Medicine"2 puts forward the argument that appropriately applied automation has the potential to aid the doctor-patient relationship and "make healthcare human again". In short, by delegating a greater portion of the highly technical aspects of medicine to Al, we have the potential to allow physicians to focus on the human aspect of their work, and to devote a larger aspect of the training of new medical professionals to developing their empathetic, patient-centered skills.
Recent applications of Al to medicine have included automatic reading of digital medical imaging, including radiology, pathology, and CT scans. Some studies have shown that Al-based analysis of highresolution pathology images can perform more accurately, as well as more quickly, than that by experts. ${ }^{3}$ Moreover, combining automated Al-based image analysis with expert reading increases the accuracy over either approach used on its own. For diagnostics, the average doctor will accumulate the experience of having seen a few thousand patients during his/her career. However, with over 700,000 physicians currently practicing in the US, computer-assisted diagnostics present the potential to leverage "experience" that is orders of magnitude higher than that of an individual practitioner. These computer-assisted diagnostics leverage both Al (in the form of deep learning from millions of case descriptions available, natural language processing to automatically interpret patient and doctor descriptions of symptoms) and crowdsourcing (allowing potentially thousands of doctors the ability to confirm or refute diagnostics within minutes or hours). Platforms such as the HumanDX app have been developed that combine both of 
these aspects of computer-assisted diagnostics. Wearable devices have been developed, such as Kardia Mobile (AliveCor, Inc.), which generate electrocardiograms on a smartphone. Al-developed algorithms interpret these EKGs on the cell phone and can alert the patient to contact the physician if a potential abnormality is detected. While these do not yet achieve the same accuracy as a hospitalbased EKG, the cheap and convenient opportunity to perform these tests on a daily basis far outweighs this limitation.

In medical research, the explosion of highthroughput genomic technologies over the last decade has benefitted from advances in data mining and machine learning, which help to process and mine data on the peta- and exa-byte scale. In cancer genomics, whole-genome and transcriptome profiles of thousands of patients have been analyzed in conjunction with treatment and outcome data in order to personalize treatment on a per-patient basis. $^{4}$

These new advances are not without their pitfalls. The majority of machine learning algorithms in use are forms of "supervised learning," in which an existing data set with known outcomes are used to generate rules for predicting the outcome of new data. As with human experience, pattern recognition is biased by previous experience. A fairly simple manifestation of these occurs with early versions of large genomic databases, which were overrepresented by samples from Caucasians. Consequently, diagnostics and treatment protocols based on derivations of these data are likely to be more effective for Caucasians than for patients of other ethnicities. Recent initiatives such as the Wellcome-Sanger Institute's UK10K project and the National Institutes of Health's "All Of Us" project have sought to remedy these deficiencies.

Avoiding bias in data sets designed for analytics whose purpose is to inform medical care is more complex than ensuring a heterogeneous genetic representation, however. The large majority of human chronic disease is believed to be caused by the interaction between genetics and exogenous factors. In this model, genetic factors predispose an individual to susceptibility to environmental factors that may trigger the disease. Consequently, the genetic landscape of a disease population with specific environmental risk factors is likely to differ from the genetic landscape of a population with the same disease but exposed to different environmental triggers. To make this concrete, imagine performing a large-scale study of the genetics of diabetes in the rural Appalachian region surrounding the institution in which I perform my research. Compared to a broader population, patients in this region would have specific characteristics in some ways that would typically be measured in such a study (for example, there would be a higher proportion of Caucasians and a higher median age), but also in ways that typically would, or could, not be measured. Rural Appalachian patients would be more likely to live in "food deserts" (giving them lower availability of fresh foods, and affecting their overall diets), and would have different profiles of exposure to air and water pollutants than an urban population. In recently adopted jargon, the rural Appalachian population would have a specific "exposome," compared to that of a more urban population. It is certainly not unrealistic to believe that these unmeasured factors could be triggers for diabetes, given a genetic predisposition to susceptibility to those triggers. Consequently, there may be genetic and genomic landscapes of diabetes that are specific to rural Appalachia, and in general, to other geographical regions.

Collecting medical data on a large-scale, making it available for research and for diagnostic and therapeutic development while ensuring appropriate data privacy and security, supporting the computational infrastructure for storing and analyzing these data, and performing these cuttingedge analyses are expensive endeavors requiring physical and human resources and expertise. As such, these projects have mostly been carried out at large medical research hospitals and institutes that are typically located in urban centers. The influence and impact of the Al revolution on medicine in the next decade is set to increase dramatically, and it is incumbent on rural medical researchers and practitioners to ensure their patients are represented in, and benefit from, this paradigm shift. 


\section{AUTHOR AFFILIATIONS}

1. Marshall University Joan C. Edwards School of Medicine, Huntington, West Virginia

\section{REFERENCES}

1. Topol EJ. High-performance medicine: the convergence of human and artificial intelligence. Nat Med. 2019;25(1):44-56.

2. Topol EJ. Deep medicine: how artificial intelligence can make healthcare human again. 1 ed. 2019: Basic Books.

3. Liu $Y$ et al. Detecting cancer metastases on giapixel pathology images. arXiv. 2017.

4. $\mathrm{Xu} \mathrm{J}$ et al. Translating cancer genomics into precision medicine with artificial intelligence: applications, challenges and future perspectives. Hum Genet. 2019;138(2):109-124. 